\title{
A comparison of oesophageal cancer between the public and private sectors in KwaZulu-Natal
}

\author{
A Lutakwa', E Loots' ${ }^{2}$ E Loots ${ }^{2}$, MT Smith ${ }^{3}$, DL Clarke ${ }^{3,4}$ \\ ${ }^{1}$ Department of Specialized Surgery Inkosi Albert Luthuli Central Hospital, Durban,University of KwaZulu-Natal Natal \\ ${ }^{2}$ Private Practice Entabeni Hospital Durban \\ ${ }^{3}$ Department of Surgery, Pietermaritzburg Hospital Complex, Pietermaritzburg, University of KwaZulu-Natal \\ ${ }^{4}$ Department of Surgery, University of the Witwatersrand, Johannesburg
}

Corresponding author: Damian Clarke (damianclar@gmail.com)

\begin{abstract}
Background: The frequency of the occurrence and type of oesophageal cancer from pathology laboratory data from the private and public sector has never been compared in KwaZulu-Natal $(\mathrm{KZN})$. This study aims to compare data from the public and private pathology services in the province.

Methods: All the malignant and pre-malignant oesophageal biopsies at the academic department of histopathology and at three private laboratories in the KZN were retrieved for comparison.

Results: Between January 2012 and December 2014, there were a total of 1087 new diagnoses of an oesophageal malignancy or pre-malignant diagnosis. There were 613 specimens from the private laboratories in KZN and 474 from IALCH. The sex distribution was (41\%) females and (59\%) males. In the private sector, the mean age was 61.6 years and in the public sector it was 62.7 years. (Range $18-75$ years) Africans comprised $63 \%$ of the total, Whites $28 \%$ and Indians $7 \%$. In the public sector $89.5 \%$ of patients were African whereas in the private sector, Africans comprised $45.7 \%$ and Whites made up $46 \%$ of the total. Eighty-eight per cent of biopsy specimens were malignant lesions of which $68 \%$ were squamous carcinoma. Squamous carcinomas accounted for $87.5 \%$ and $54 \%$ of all lesions in the public and private sectors respectively. Pre-malignant lesions accounted for $5 \%$ and $23 \%$ of diagnoses in the public and private sectors respectively. Pre-malignant lesions in the public sector showed mainly dysplastic squamous cell dysplasia whereas dysplastic Barrett's oesophagus lesions accounted for the majority in the private sector

Conclusion: Oesophageal cancer is a major public health problem in KZN. Although squamous carcinoma predominates, adenocarcinoma is a significant problem in the White population. It would appear that surveillance for oesophageal adenocarcinoma is taking place in the private sector albeit in a non-systematic or coordinated fashion. This does not appear to be the case in the state sector.
\end{abstract}

S Afr J Surg 2019;57(2)

http://dx.doi.org/10.17159/2078-5151/2019/v57n2a2871

\section{Introduction}

Oesophageal cancer in South Africa is a neglected public health problem. ${ }^{1,2}$ It is one of the commonest cancers in males and has a poor prognosis, yet there has been very little research on the topic and almost no attempt to develop a comprehensive response to the disease over the last two decades. ${ }^{1,2}$ In South Africa, there is a national cancer registry which collates national histology data, and a major population-based cancer registry based in the Eastern Cape Province. ${ }^{3,4}$ Both of these attempt to provide a current overview of the burden of cancer in South Africa, however both registries are beset with a number of problems which tend to limit their usefulness. South Africa has a two-tier health system, with the government or public sector serving
$80 \%$ of the population and the private sector serving the remaining $20 \%$. Health care expenditure in the private sector accounts for over half the nation's total expenditure on health care. ${ }^{5}$ National Health Insurance (NHI) is an attempt to address this inequity and to improve health care outcomes for the population. In attempting to formulate health care reform, there is a need to develop an overarching and unitary strategic vision and plan, to deal comprehensively with the challenges facing the health care system. ${ }^{5}$ Oesophageal cancer in particular and gastrointestinal malignancy in general, represent just such a challenge. Currently, there is very little research comparing diseases and outcomes in the private and public sectors and this makes it difficult to formulate policy options and strategies. The way private practice is structured with its many independent practitioners makes it very difficult 
to perform surgical audit. There are a number of potential sources of data and these include health insurance company data and individual hospital group billing data. One potential source of information which may allow for comparisons between the two sectors is pathology data. This study uses pathology data from the three main private pathology services in KwaZulu-Natal (KZN) Province and compares their data set of all patients with a malignant and pre-malignant oesophageal lesion with the histology data set from the major state pathology unit in the province, at Inkosi Albert Luthuli Central Hospital (IALCH). It is hoped that this will provide a province-wide overview of the problem and will provide a unified data set covering both private and government sector patients.

\section{Setting}

The Province of KwaZulu-Natal is situated on the south eastern seaboard of South Africa. It covers an area of 94361 square kilometres. According to the most recent census there are 10267300 people in KZN. Approximately $34 \%$ of the population reside in eThekwini Metro which incorporates the major port city of Durban. The racial profile of the province is as follows: Black Africans (86.8\%), Indian (7.4\%), White (4.2\%) and Mixed Race (1.4\%). There is a twotier health service in South Africa. The private sector provides care for about $20 \%$ of the population. The state sector provides care for the remaining $80 \%$. All histology in the state sector is reviewed by the Department of Histopathology based at IALCH. There are three main private laboratories which provide pathology services to the private sector.

\section{Methods}

All malignant and pre-malignant oesophageal biopsies at the three major private pathology laboratories in $\mathrm{KZN}$ and the Department of Histopathology at IALCH were retrieved and entered onto an Excel spreadsheet for comparison. Categorical data was summarised by frequency and per cent. Subgroup comparisons by type of cancer and malignancy were compared by demographic risk factors using chi square tests. Odds ratios, 95\% confidence limits and p-values are reported. Data was analysed in Stata V13.1.

\section{Results}

Between January 2012 and December 2014, one thousand one hundred and twenty seven (1127) patients were identified of which, 31 had a benign oesophageal pathology (oesophageal candidiasis) and were excluded. Six patients who had an unknown diagnosis and three duplicate entries were also excluded. This left a total of 1087 new diagnoses of an oesophageal malignancy or a pre-malignant oesophageal lesion for the two-year period. Figure1 shows the patient sample. There were 613 specimens from the private laboratories and 474 from IALCH. There were 728 squamous carcinomas (67\%), 160 adenocarcinomas (15\%), 167 premalignant lesions (15\%) and 32 miscellaneous lesions (3\%). (Table 1). The sex distribution was (41\%) females and $(59 \%)$ males. The mean age in the private sector, was 61.6 years and in the public sector 62.7 years. The age range was $18-75$ years. Africans comprised $63 \%$ of the total, Whites $28 \%$ and Indians $7 \%$. The racial distribution varied across public and private centers. In the public sector $89.5 \%$ of patients were African. In the private sector, Africans comprised only $45.7 \%$ of the total and Whites made up the remaining $46 \%$. The breakdown of the different histological diagnoses against private or public sector is provided in Table 1. Malignant lesions made up $88 \%$ of all biopsy specimens and pre-malignant lesions $12 \%$. The majority of malignant diagnoses $(68 \%)$ were squamous carcinoma. In the state sector the vast majority of tumours were squamous $(87.5 \%)$. In the private sector this predominance was less marked and only $54 \%$ of all lesions were squamous cell carcinomas. The

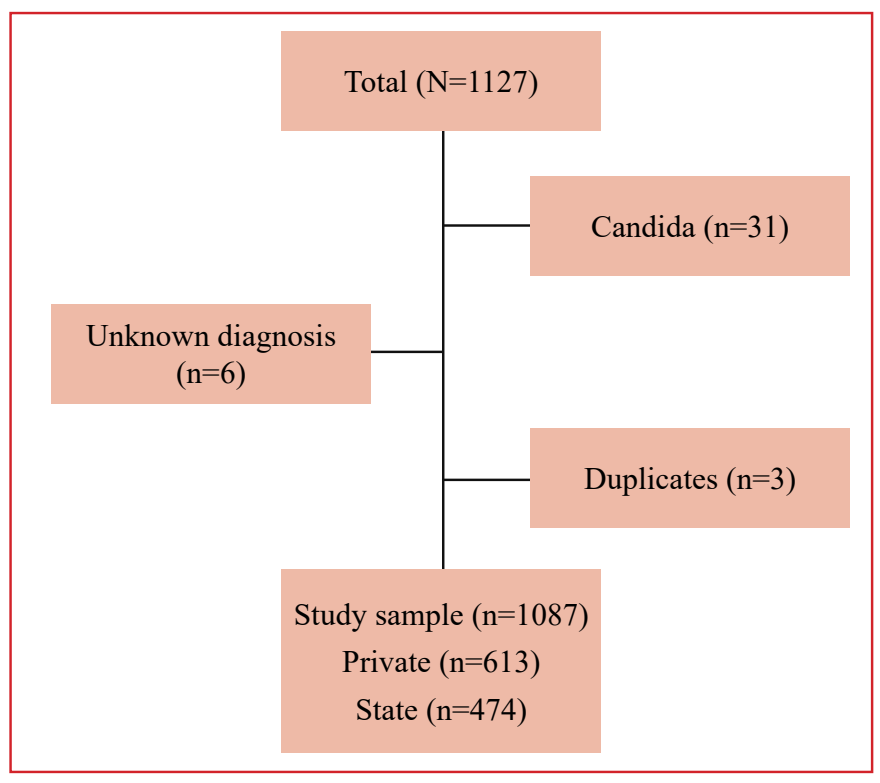

Figure 1. Flow diagram of the study population $(n=1127)$ and study sample $(n=1082)$

Table 1. Cancer Type

\begin{tabular}{lccccc} 
Table 1. Cancer Type & Private N & Private $\%$ & Public $\mathbf{~}$ & Public \% & Total \\
\hline Cancer Type & 330 & 53.9 & 398 & 83.8 & 728 \\
\hline Squamous & 143 & 23.4 & 17 & 3.6 & 160 \\
Adenocarcinoma & 136 & 22.2 & 31 & 6.61 & 167 \\
Pre-malignant & 4 & 0.5 & 28 & 5.97 & 32 \\
Other & 613 & 100 & 474 & 100 & 1087
\end{tabular}




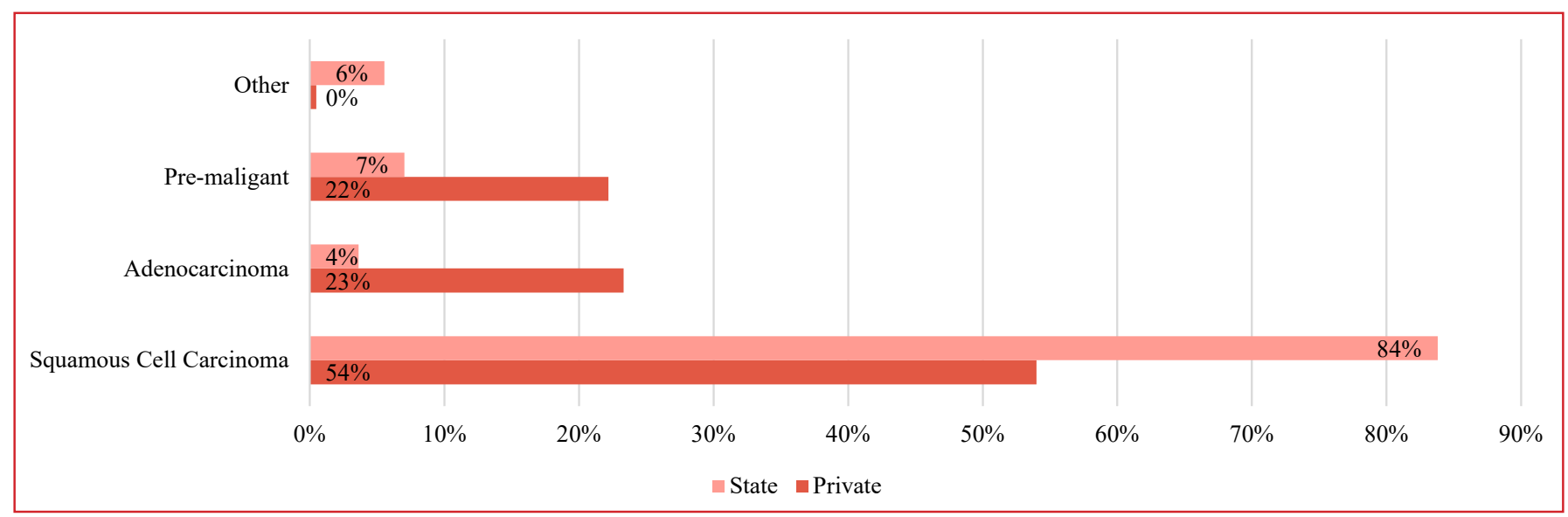

Figure 2 : Breakdown of pathological diagnoses between public and private sectors

incidence of squamous carcinoma was significantly higher in African patients (OR 15.5, 95\% CI 10.3-23.5 p < 0.001), in female patients (OR 3.3, 95\% CI 2.2-5.0 p < 0.001) and in patients in the public sector (OR 10.10 95\% CI 5.9-16.9) $\mathrm{p}<0.001)$. In the private sector $23 \%$ of specimens were premalignant lesions. These pre-malignant lesions were Barrett's oesophagus lesions. In the state sector only five per cent of diagnoses were pre-malignant. The pre-malignant lesions in the state sector were all squamous in nature. African patients were significantly more likely to have a malignant diagnosis rather than a pre-malignant diagnosis in comparison to White patients (OR 8.6 95\% CI 5.8-12.6 p < 0.001). This difference persisted when only the private sector patients were considered (OR 8.6 95\% CI 5.8-12.6 p < 0.001). Based on an average of 529 new cases a year and a population of 10.2 million people, the rate of cancer of the oesophagus in $\mathrm{KZN}$ is 5.18 per 100000 people.

\section{Discussion}

The National Cancer Registry of South Africa (NCRSA) records pathology diagnoses of cancer, from laboratories around the country. ${ }^{3,4}$ According to the registry, oesophageal cancer is the sixth most common cancer in men and the eight most common cancer in women. The reported incidence in men is 7/100 000 and in women 4/100 000 . The only population-based cancer registry is in the Eastern Cape Province. This reports a much higher incidence of oesophageal cancer of 23.2/100 000 in men and 14.5/100 000 in women for the period 2008-2012.,4 This excessively high incidence in the Eastern Cape has long been noted and is an ongoing area of investigation. . $^{3,4}$ Our estimated incidence based on histology specimens is 5/100 000 for the Province of KwaZulu-Natal. This is in keeping with the figures from NCRSA and is lower than that in the neighbouring province of the Eastern Cape. However, this may be an underestimation as the figure is based on confirmed histology, and many patients will not have a histological diagnosis established during their care. Based on the absolute numbers, oesophageal cancer is a major public health problem in KZN and a strong case can be made for centralising the management of oesophageal cancer at a center capable of providing multi-modal management. Generally, less than $10 \%$ of these lesions are amenable to curative resection and this translates into between 25 and 50 patients a year requiring an oesophageal resection. With an average operative time in the range of four hours and with the requirement for postoperative high care or ICU, this has major implications for cancer services in KZN. ${ }^{1,2}$

It would appear that the major form of the disease remains squamous cancer and this overwhelmingly affects African patients and is more common in female patients. This female predominance is a new finding which has been previously commented upon by our group. ${ }^{6}$ Our current data challenges the view that oesophageal cancer is a predominantly male disease in KZN province. Ongoing audit is needed to establish the veracity of this observation. This paper shows how the demographics of the private sector are changing as more African patients access private care. It is important to develop an understanding of the demographics and spectrum of diseases in the private sector as there is a drive to integrate the sectors and their funding. It may well be in the future that the National Health Insurance (NHI) attempts to coordinate, management of complex gastrointestinal cancers and to do this an accurate picture of the spectrum of disease in both sectors is necessary. ${ }^{5}$ Having said this, it is apparent that adenocarcinoma of the oesophagus predominantly affects White and Indian patients and that most of the burden of adenocarcinoma of the oesophagus is treated in the private sector. ${ }^{6-9}$

Pre-malignant biopsy results were predominantly confined to the private practice cohort and were overwhelmingly Barrett's oesophagus, which is a precursor of adenocarcinoma. This suggests that targeted screening is being performed in the private sector and that there is an attempt at surveillance in this sector. It is of concern that there was a low rate of pre-malignant biopsies in the state sector cohort. This suggests that there is minimal screening or surveillance being undertaken in the state sector, at least in the highrisk population groups. There is increasing evidence that squamous oesophageal cancer like adenocarcinoma of the oesophagus, has premalignant lesions..$^{6-9}$ These may well be related to exposure to Human Papilloma Virus (HPV) as is 
the case with a number of other squamous cancers. A better understanding of these lesions and their relationship to the development of squamous oesophageal cancer may allow for earlier detection of these cancers. It would seem that premalignant lesions are not being detected in the state sector in significant volume. Currently there appears to be a lack of awareness about premalignant lesions of the oesophagus and inadequate access to upper endoscopy services in the state sector. ${ }^{10}$ These deficits need to be addressed as part of NHI and future strategic health plans.

\section{Conclusion}

Oesophageal cancer is a major public health problem in KZN and the large number of new diagnoses in a single year suggests that a dedicated program based at a major center capable of delivering multi-modal treatment is necessary. Although squamous carcinoma predominates, adenocarcinoma is a problem in the White population. It would appear that there is a great deal of active surveillance for oesophageal cancer taking place in the private sector albeit in a non-systematic and uncoordinated fashion. This does not appear to be happening in the state sector and this deficit needs to be addressed.

\section{Conflicts of Interest}

The authors have no conflicts of interest to declare.

No external or internal funding was utilised to generate this work

\section{REFERENCES}

1. Loots E, Sartorius B, Madiba TE, Mulder CJ, Clarke DL. Is Clinical Research in Oesophageal Cancer in South
Africa in Crisis? A Systematic Review. World J Surg. 2017 Mar;41(3):810-6. doi: 10.1007/s00268-016-3778-5. Review

2. Govender M, Ferndale L, Clarke DL. Oesophageal cancer in South Africa: The long timeline from onset of symptoms to definitive management. S Afr J Oncol. 2017;1(0), a6. h ps://doi. org/10.4102/sajo.v1i0.6

3. Somdyala NI, Parkin DM, Sithole N, Bradshaw D. Trends in cancer incidence in rural Eastern Cape Province; South Africa, 1998-2012. Int J Cancer. 2015 Mar 1;136(5):E470-4. doi: 10.1002/ijc.29224. Epub 2014 Oct 3.

4. (http://www. afcrn.org/membership/members/87-ncrsa). (Accessed June 2018)

5. McIntyre D. Private sector involvement in funding and providing health services in South Africa: Implications for equity and access to health care. EQUINET, Harare: Health Economics Unit, 2010.

6. Loots E, Sartorius B, Madiba TE, Mulder CJJ, Clarke DL. Oesophageal squamous cell cancer in a South African tertiary hospital: a risk factor and presentation analysis. S Afr J Surg. 2017;55(3).

7. Alema ON, Iva B. Cancer of the esophagus: histopathological sub-types in northern Uganda. Afr Health Sci. 2014 Mar;14(1):17-21. doi: 10.4314/ahs.v14i1.4

8. Henry MA, Lerco MM, Ribeiro PW, Rodrigues MA. Epidemiological features of oesophageal cancer. Squamous cell carcinoma versus adenocarcinoma. Acta Cir Bras. 2014 Jun;29(6):389-93.

9. Safaee A, Fatemi SR, Moghimi-Dehkordi B, Ghiasi S, Zali MR. Epidemiology and histopathological features of oesophageal cancer. East Afr J Public Health. 2012 Mar;9(1):7-9.

10. Loots E, Clarke DL, Newton K, Mulder CJ. Endoscopy services in KwaZulu-Natal Province, South Africa, are insufficient for the burden of disease: Is patient care compromised? S Afr Med J. 2017 Oct 31;107(11):1022-5. doi: 10.7196/SAMJ.2017. v107i11.12484 